\title{
ARTICLE
}

\section{Early life alcohol exposure primes hypothalamic microglia to later-life hypersensitivity to immune stress: possible epigenetic mechanism}

\author{
Lucy G. Chastain ${ }^{1}$, Tina Franklin ${ }^{1}$, Omkaram Gangisetty ${ }^{1}$, Miguel A. Cabrera ${ }^{1,2}$, Sayani Mukherjee ${ }^{1}$, Pallavi Shrivastava ${ }^{1}$,
} Shaima Jabbar ${ }^{1,2}$ and Dipak K. Sarkar ${ }^{1}$

Growing evidence has shown that developmental alcohol exposure induces central nervous system inflammation and microglia activation, which may contribute to long-term health conditions, such as fetal alcohol spectrum disorders. These studies sought to investigate whether neonatal alcohol exposure during postnatal days (PND) 2-6 in rats (third trimester human equivalent) leads to long-term disruption of the neuroimmune response by microglia. Exposure to neonatal alcohol resulted in acute increases in activation and inflammatory gene expression in hypothalamic microglia including tumor necrosis factor alpha (TNF-a) and interleukin 6 (IL-6). Adults with neonatal alcohol pre-exposure (alcohol fed; AF) animals showed an exaggerated peripheral stress hormonal response to an immune challenge (lipopolysaccharides; LPS). In addition, there were significantly more microglia present in the hypothalamus of adult AF animals, and their hypothalamic microglia showed more cluster of differentiation molecule $11 \mathrm{~b}$ (Cd11b) activation, TNF-a expression, and IL-6 expression in response to LPS. Interestingly, blocking microglia activation with minocycline treatment during PND 2-6 alcohol exposure ameliorated the hormonal and microglial hypersensitivity to LPS in AF adult animals. Investigation of possible epigenetic programming mechanisms by alcohol revealed neonatal alcohol decreased several repressive regulators of transcription in hypothalamic microglia, while concomitantly increasing histone H3 acetyl lysine 9 (H3K9ac) enrichment at TNF-a and IL-6 promoter regions. Importantly, adult hypothalamic microglia from AF animals showed enduring increases in H3K9ac enrichment of TNF- $a$ and IL- 6 promoters both at baseline and after LPS exposure, suggesting a possible epigenetic mechanism for the long-term immune disruption due to hypothalamic microglial priming.

Neuropsychopharmacology (2019) 44:1579-1588; https://doi.org/10.1038/s41386-019-0326-7

\section{INTRODUCTION}

Despite public awareness about the risks of drinking during pregnancy, recent studies have shown that health problems due to fetal exposure to alcohol are still common in the US [1] and globally [2]. Fetal alcohol exposure may result in a variety of health problems during childhood and adolescence, which may include facial and cranial dysmorphologies, brain abnormalities, neurological defects, cognitive deficits, delayed development, endocrine abnormalities, and problems with mood and anxiety which are generally called fetal alcohol spectrum disorders (FASDs) [3, 4]. Many of the symptoms of FASDs can persist through adulthood [5]. Animal models indicate the hypothalamus and the hypothalamic-pituitary-adrenal (HPA) axis, or stress axis, are especially sensitive to the long-term effects of developmental alcohol $[6,7]$. In addition to the hallmark neurological and psychiatric symptoms, clinical studies also report people with FASDs have higher incidences of infections [8], which suggest immune system disruption in FASDs $[6,7]$.

Microglia are one type of resident immune cell in the central nervous system. Under normal physiological conditions, microglia take on a surveillance and support role for neuronal activity, but upon central nervous system insult, microglia become reactive and may release pro-inflammatory cytokines and chemokines contributing to neuroinflammation and neurotoxicity [9]. Many studies have provided strong evidence that pathological alcohol consumption exerts a pro-inflammatory effect on the neuroimmune system due to microglia activation [10, 11]. Clinical studies of post-mortem brain tissue from people with alcohol use disorders (AUDs) show increased pro-inflammatory and microglial markers [12] alongside neural damage [13]. Studies in animal models also show high dose alcohol exposure increases proinflammatory factors, microglia activation, and neurotoxicity in several brain regions in both adult animals and developing animals $[10,11,14]$. Studies utilizing cultured primary microglia and beta-endorphin (BEP) producing cells from fetal rat hypothalamus, have shown that microglia play a direct neurotoxic role in killing hypothalamic neurons after high dose alcohol exposure through the release of TNF- $\alpha$ and IL- $6[15,16]$.

Although data from our lab and others suggest that alcohol exposure may cause microglia to become pro-inflammatory and to potentiate alcohol's neurotoxic effects, the role of microglia in the long-term detrimental effects of developmental alcohol are not well understood. Some studies utilizing neonatal or fetal alcohol exposure models in rodents find only transient changes in

\footnotetext{
${ }^{1}$ The Endocrine Program, Department of Animal Sciences, Rutgers, The State University of New Jersey, New Brunswick, NJ, USA and ${ }^{2}$ Endocrinology and Animal Biosciences Graduate Program, Rutgers, The State University of New Jersey, New Brunswick, NJ, USA

Correspondence: Dipak K. Sarkar (sarkar@aesop.rutgers.edu)
}

Received: 20 August 2018 Revised: 16 December 2018 Accepted: 11 January 2019

Published online: 30 January 2019 
a

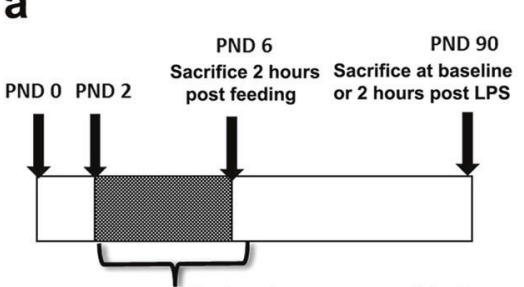

Alcohol-fed $2.5 \mathrm{~g} / \mathrm{kg} /$ day (11.34\% alcohol) (AF) Alcohol-fed + minocycline (AF+M) Pair-fed isocaloric milk formula (PF) Ad lib-fed suckle controls (AD)

d

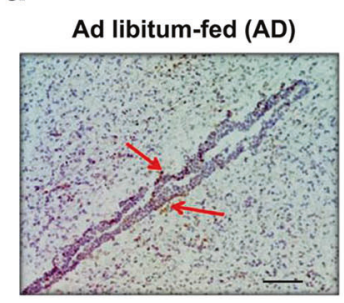

e

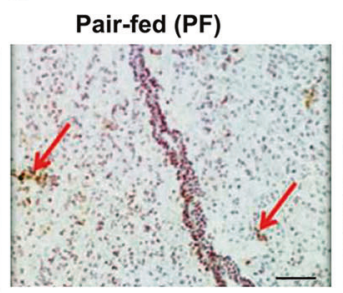

b

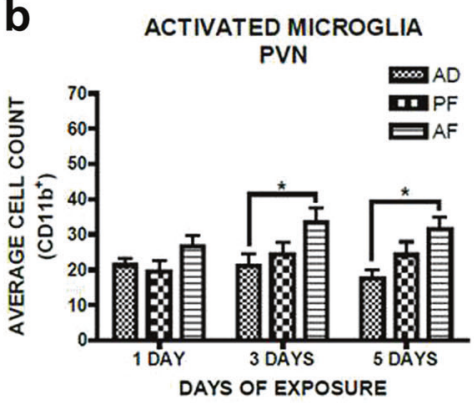

f

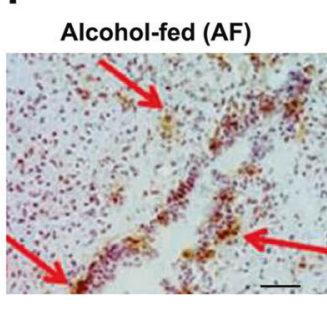

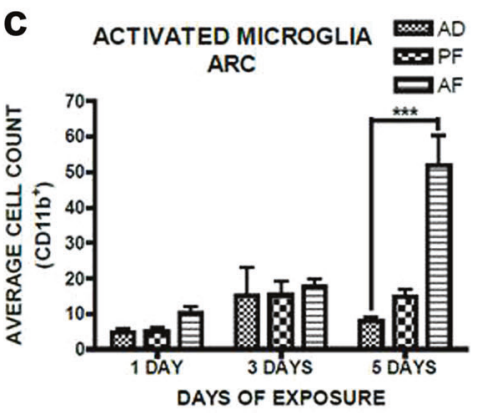

g

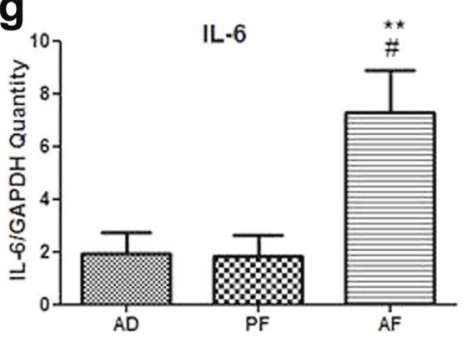

h

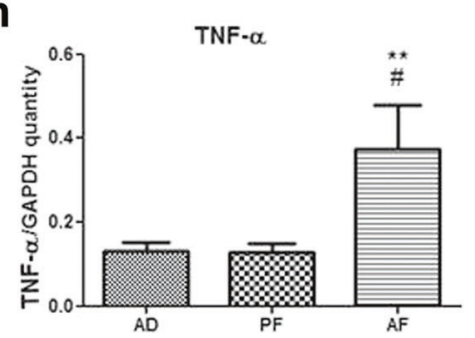

i

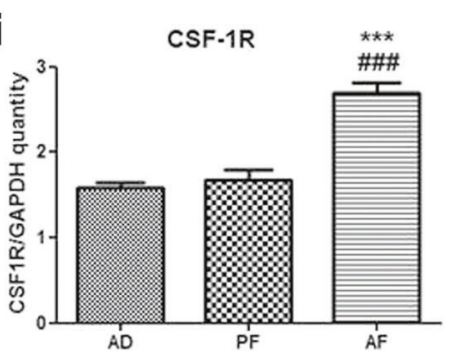

j

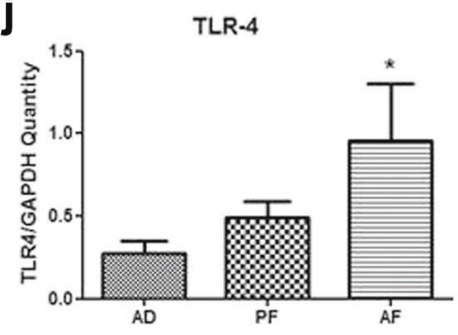

Fig. 1 Neonatal alcohol activates hypothalamic microglial inflammation in vivo. a Design of in vivo experiments. Immunohistochemical staining of reactive microglial marker Cd11b in paraventricular nucleus (PVN) and arcuate nucleus (ARC) of alcohol-fed (AF), pair-fed (PF), and ad libitum-fed (AD) pups $2 \mathrm{~h}$ post feeding. AF pups showed increased Cd11b+ cell count in the PVN (b) and arcuate (ARC) nucleus (c) of the hypothalamus after 5 days of alcohol exposure compared to AD pups ( $n=4-6 /$ treatment group). Representative immunohistochemistry images of $C d 11 b+$ staining in PND 6 of AD (d), PF (e), and AF (f) pups. Scale bars in these figures are $100 \mu$ m/each. AF pups showed increased microglial expression of IL-6 (g), TNF- $\alpha(\mathbf{h})$, CSF1R (i), and TLR4 (j) compared to PF and AD pups $(n=4-6 /$ treatment group). Data are mean \pm

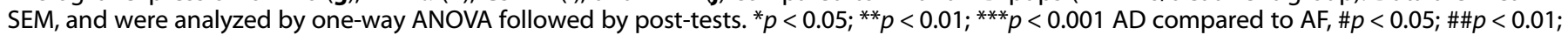
$\# \#$ \# $<0.001 \mathrm{PF}$ compared to $\mathrm{AF}$

microglia activation and pro-inflammatory cytokine expression in brain tissue after alcohol exposure $[17,18]$. In contrast, other studies reported lasting upregulation of inflammatory factors at postnatal day 28 following neonatal alcohol exposure [19] and at adulthood following adolescent alcohol exposure [20, 21]. Several studies suggest microglia possess the ability to "remember" past activation events and alter their response to later inflammatory insults in a phenomenon called priming. Microglia may be primed by early life infection [22], brain injury [23], aging and stress [24], or neurodegenerative disease [25]. The mechanisms for microglia priming are not understood, but as microglia activation and immune response are tightly regulated by epigenetic factors [26], it is possible that epigenetic programming may be a potential mechanism. Developmental alcohol is known to affect epigenetic mechanisms at the level of methylation and histone modifications $[27,28]$, but most studies to date have utilized brain tissue homogenates, thus, making conclusions about alcohol's effects on specific cell types, like microglia, impossible.

The HPA axis of the developing brain is especially susceptible to developmental programming by stressors during the second and third trimester periods of pregnancy $[29,30]$. In rodents, the first two weeks of life corresponds to the third trimester in humans, and recent studies have utilized this time period for investigation of the mechanisms of long-lasting developmental programming $[31,32]$. In our studies, we address the question of whether early life alcohol primes hypothalamic microglia and the HPA axis to alter responsivity later in life and whether this phenomenon may have an epigenetic mechanism. To address these questions, we utilized the postnatal day 2-6 period in rats, an established fetal alcohol use disorder model to mimic high alcohol exposure during human third trimester equivalent [16].

\section{MATERIALS AND METHODS}

Animals

Adult Sprague-Dawley rats were purchased from Charles River Laboratories (Wilmington, MA). Animls were housed under standard lighting conditions ( $12 \mathrm{~h}$ on; $12 \mathrm{~h}$ off) and had access to rodent chow and water ad libitum (AD). These rats were bred in our colony to generate neonatal animals and adult animals for use in these studies. Hypothalami from PND 1 old rat pups were used to generate primary microglia culture. Animal surgery and care were performed in accordance with institutional guidelines and complied with NIH policy. The animal protocol was approved by the Rutgers Animal Care and Facilities Committee.

Experimental design for in vivo experiments

See Fig. 1a for the in vivo experimental design. Offspring were assigned to one of four groups: (1) alcohol fed (AF) animals that were fed by oral gavage a milk formula containing $11.34 \%$ ethanol 
$(\mathrm{ETOH})(\mathrm{vol} / \mathrm{vol})$ and daily s.c. treatment of saline $(50 \mu \mathrm{l}) 1 \mathrm{~h}$ before alcohol feeding, (2) AF animals that also received daily s.c. treatment with minocycline $(45 \mu \mathrm{g} / \mathrm{kg}) 1 \mathrm{~h}$ before alcohol feeding $(\mathrm{AF}+\mathrm{M})$, (3) pair fed (PF) animals that were fed isocaloric formula only and injected daily saline $(50 \mu \mathrm{l})$ and (4) AD animals that were left in the litter with the mother. The daily alcohol dose was $2.5 \mathrm{~g} /$ $\mathrm{kg}$, and produced mean blood alcohol concentrations (BACs) of $150.1 \mathrm{mg} / \mathrm{dL} 2 \mathrm{~h}$ after the last feeding, concentrations which resemble binge drinking levels in humans. Feedings were given twice daily at 10:00 a.m. and 12:00 p.m. for 5 days on PND 2-6. The alcohol dosage and feeding protocol has been shown to produce significant microglial activation and neuroinflammation in PND 6 rat pups [16]. In addition, our minocycline dose was chosen based on our previous work [16], showing minocycline $(45 \mu \mathrm{g} / \mathrm{kg})$ sufficiently blocks alcohol-induced BEP cell neurotoxicity in PND 6 pups. On PND 6, a group of animals were sacrificed at $2 \mathrm{~h}$ after the last feeding. Preliminary studies did not indicate any sex differences in microglial response to alcohol at this time point [16], so both male and female subjects were used in our studies. Brains were collected and frozen at $-80^{\circ} \mathrm{C}$ for later use for immunohistochemical staining of microglia or microglia were isolated from dissected mediobasal hypothalamus $(\mathrm{MBH}$; the mediobasal portion of the hypothalamus extended $\sim 1 \mathrm{~mm}$ rostral to the optic chiasma and just caudal to the mammillary bodies, laterally to the hypothalamic sulci, and dorsally to $2 \mathrm{~mm}$ deep) for measurement of RNA, protein, DNA methylation, or for chromatin immunoprecipitation (ChIP).

Animals used for the adult studies were assigned to the four treatment groups as described above $(A F, A F+M, P F, A D)$, and after PND 2-6 treatment, were kept with the dam until PND 23 when they were then weaned and housed 2-3 animals/cage until they were utilized at PND 90. Males only were used for these adult studies. Follow-up studies utilizing females are currently underway in our lab to investigate the effects of neonatal alcohol on adult females. Adult animals were either sacrificed for basal measurements or were challenged with lipopolysaccharide (LPS) $(100 \mu \mathrm{g} /$ $\mathrm{kg}$ body weight) injected i.p., and sacrificed $2 \mathrm{~h}$ later. Previous studies in our laboratory have shown that this dose of LPS effectively stimulates the hormonal stress axis (42). For measurement of stress hormones, baseline tail blood was collected in the morning (9:00-11:00 a.m.) and trunk blood was collected $2 \mathrm{~h}$ after LPS (a timepoint where we find maximal stress hormones responses to LPS). For immunohistochemical staining of microglia, brains were collected at baseline and post LPS and frozen at $-80^{\circ} \mathrm{C}$ until use. In addition, microglia were isolated from dissected $\mathrm{MBH}$ for measurement of RNA, protein, DNA methylation, or ChIP at baseline and post LPS.

Primary microglia cell culture

Microglia cell culture was prepared from $\mathrm{MBH}$ from postnatal day 1 rat pups from both sexes using the method published by us previously $[15,16]$. (See Supplementary Materials and Methods for details.) To assess the effect of acute alcohol treatment on microglia cell cultures, microglia cells were treated with $50 \mathrm{mM}$ $\mathrm{ETOH}$-treated for $24 \mathrm{~h}$ or vehicle (control). Alcohol evaporation was prevented by culturing cells in slightly close $25 \mathrm{~mm}$ flask and "refreshing" alcohol-treated cultures at the $12 \mathrm{~h}$ timepoint $(24 \mathrm{~h}$ total time for treatment) with fresh alcohol. After $24 \mathrm{~h}$, cells were collected and used for RNA measurements, ChIP, or protein measurements. The $50 \mathrm{mM}$ ETOH dose resembles binge drinking levels, and has also been shown to produce microglial activation, inflammation, and neurotoxicity in hypothalamic BEP-producing neurons $[15,16]$.

In vivo microglia isolation

Microglia were purified from freshly dissected $\mathrm{MBH}$ tissue from PND 6 pups and adult rats by an Optiprep (Sigma-Aldrich) density gradient using methods described by us previously [16]. (See Supplemental Materials and Methods for details.) Three hypothalami from PND 6 pups were pooled to generate samples for the pup experiments and two hypothalami from adult rats were pooled to generate samples for the adult experiments. Cells were used for RNA measurements, ChIP, or protein measurements immediately or stored at $-80^{\circ} \mathrm{C}$ until use.

Measurement of gene expression by quantitative reverse transcription polymerase chain reaction (RT-PCR)

Gene expression of mRNA encoding IL-6, TNF- $a$, CSF-1R, TLR-4, MCP-1, and IL-1B in microglia was measured by quantitative RTPCR using a SYBR green assay (Thermo Fisher Scientific; 16). See Table S1 and the Supplemental Materials and Methods for details.

Immunocytochemistry and flow cytometry

Protein measurements were made on cultured microglia and in vivo isolated microglia by immunocytochemical fluorescent staining and flow cytometry detection of immunofluorescence as previously described by us [16]. See the Supplemental Materials and Methods for details.

Immunohistochemical staining of microglia in tissue sections Serial coronal sections of frozen brains ( $20 \mu \mathrm{m}$ thickness) ranging from the paraventricular nucleus (PVN) and arcuate nucleus (ARC) were stained for total microglial population using IBA1 as a specific marker (Wako, Richmond, VA) or reactive microglia using Cd11b as a marker (clone OX32) (AbD Serotec Bio-Rad, Hercules, CA) [16]. Staining was visualized using a Nikon TE 2000 inverted microscope (Nikon Instruments Inc., Melville, NY). Total Cd11b+ and IBA-1+ cells as identified by positive staining were counted in PVN and ARC regions as identified by adult [33] and neonatal [34] rat brain atlases. Mean total $\mathrm{Cd} 11 \mathrm{~b}+$ and IBA-1+ cell counts were obtained from three sections per brain for PVN, and 10 sections per brain for the ARC. See the Supplemental Materials and Methods for details.

\section{Global DNA methylation quantification}

Total DNA was isolated from in vivo-isolated microglia using the QIAamp DNA Micro Kit (Qiagen, Germantown, MD) and from cultured microglia using the DNEasy Blood and Tissue Kit (Qiagen). Global DNA methylation levels were quantified from $100 \mathrm{ng}$ DNA using the MethylFlash Methylated DNA Quantification kit (Epigentek, Farmingdale, NY), an antibody-based ELISA-like kit for quantitative detection of global levels of methylated DNA. The assay was conducted according to the manufacturer's instructions and \% 5-methylcytosine $(5-\mathrm{mc})$ levels were calculated from the 5$\mathrm{mc}$ standard curve provided by the assay kit.

Stress hormone measurement after immune challenge The stress hormones corticosterone (CORT) and adrenocorticotropic hormone (ACTH) levels in plasma were measured using rodent CORT ELISA (Immuno-Biologic Labs, Minneapolis, MN) and rodent ACTH ELISA (Phoenix Pharmaceuticals, Burlingame, CA).

\section{Chromatin Immunoprecipitation}

Isolated in vivo microglia and cultured microglia were used for ChIP of histone $\mathrm{H} 3$ acetyl lysine 9 (H3K9ac). Alcohol-induced H3K9ac enrichment at IL-6 and TNF-a promoter regions were determined by ChIP using $\mathrm{H} 3 \mathrm{~K} 9 \mathrm{ac}$ antibody and measure promoter-specific amplification using real-time PCR. ChIP was performed using rabbit polyclonal anti-acetyl-Histone H3 (Lys9) (Millipore, Billerica, MA) and the ChIP Assay Kit (Cat \# 17-295, Millipore) according to the manufacturer's instructions. See Table S1 and the Supplemental Materials and Methods for details. 

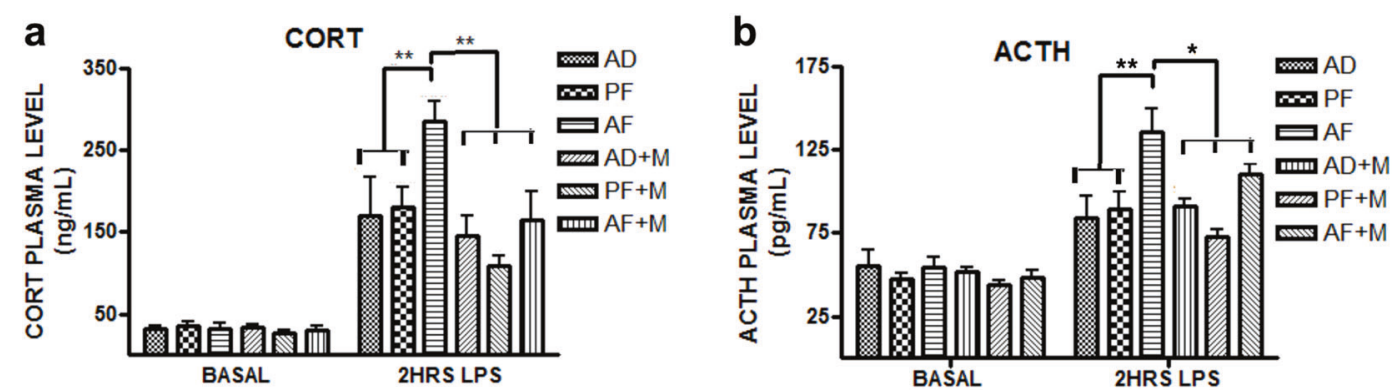

C

ACTIVATED MICROGLIA ARC

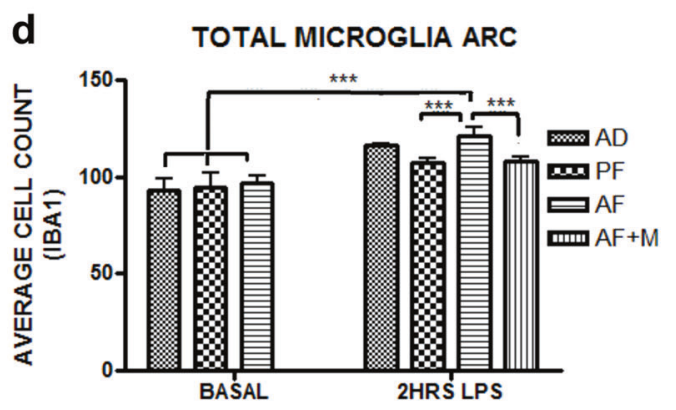

e
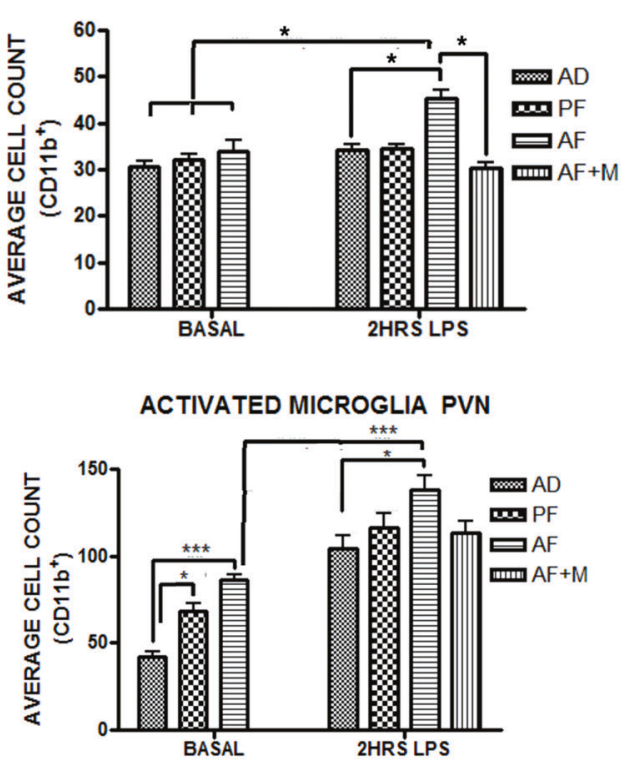

$9 \quad$ Ad libitum-fed (AD) $h \quad$ Pair-fed (PF)
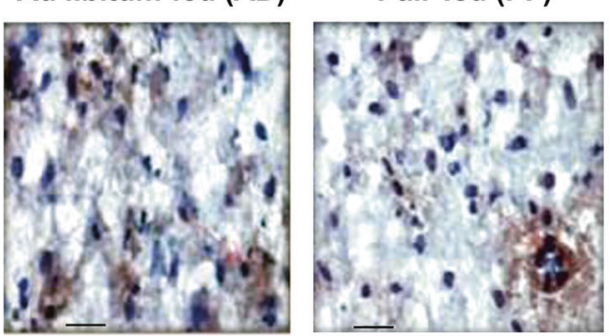

k

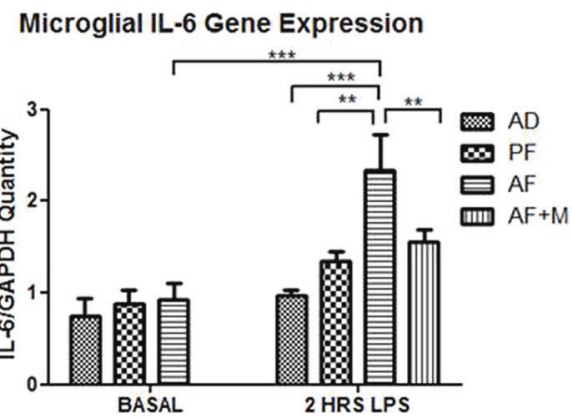

f
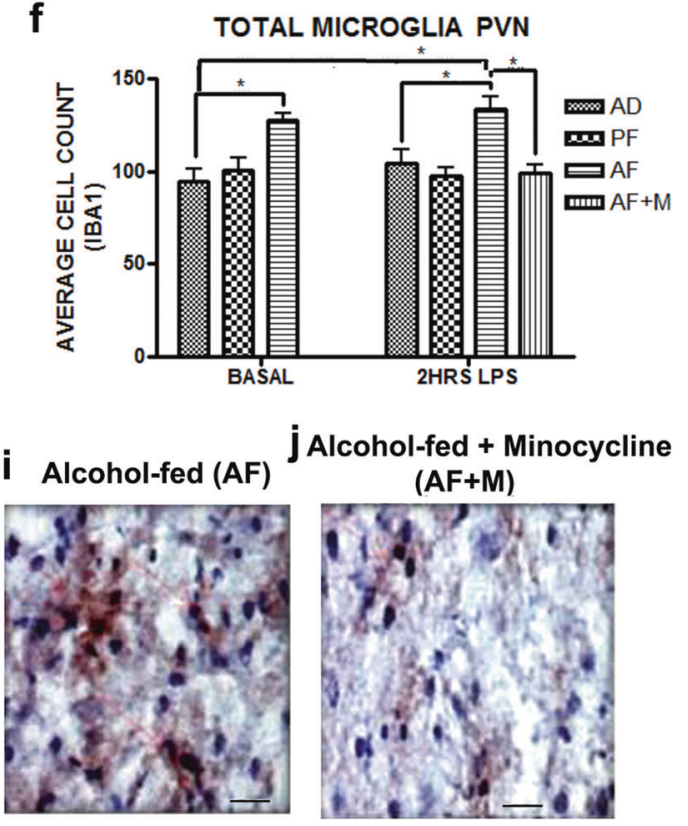

I

Microglial TNF- $\alpha$ Gene Expression

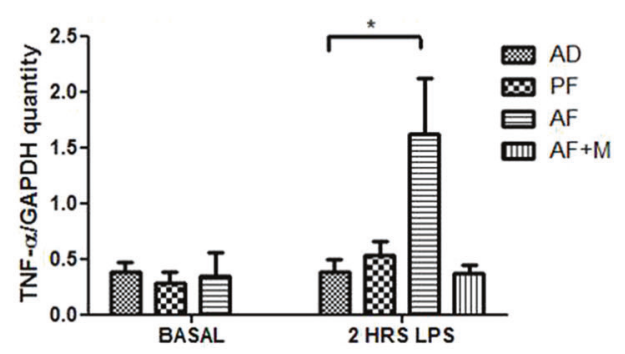

Statistical analyses

The data in the figures are presented as mean \pm SEM. One-way ANOVA was used to analyze differences between treatment groups, followed by Newman-Keuls post-tests. In cases where variances differed between treatment groups, the Kruskal-Wallis

test was utilized, followed by Dunn's multiple comparison posttest. Two-way ANOVA was used for multiple comparisons, followed by Bonferroni post-tests. Student's $t$-test was used to analyze differences between two treatment groups. $P \leq 0.05$ was considered significant. 
Fig. 2 Neonatal alcohol pre-exposure results in hyper-response to immune stress, which is normalized by minocycline. The long-lasting hormonal and microglial effects of neonatal alcohol in adult animals at baseline and in response to an i.p. LPS challenge (0.1 mg/kg) were examined. Adult AF animals showed no differences in basal levels of plasma CORT (a) and ACTH (b), but showed an exaggerated CORT and ACTH response to an LPS challenge as compared to AD animals, which was ameliorated by concurrent PND 2-6 minocycline treatment (AF $+\mathrm{M})(n=5-6 /$ treatment group). Total number of microglia (as identified by IBA $1+$ staining) in the ARC nucleus of the hypothalamus was not significantly different in AF animals (d), but AF animals had significantly increased activated microglia (Cd11b+) in the ARC which was diminished by concurrent administration of minocycline $(A F+M)(c)(n=5 /$ treatment group). Increased basal and LPS-induced total number of microglia (e) and activated microglia ( $\mathbf{f})$ was found in the PVN region of the hypothalamus $(n=5 /$ treatment group). LPS-induced increase in AF number of microglia was diminished by concurrent PND6 minocycline treatment $(A F+M)$. Representative immunohistochemistry images of Cd11b+ staining in AD (g), PF (h), AF (i), and AF Minocycline (j) in the PVN post LPS. Scale bars in these figures are $400 \mu \mathrm{m} /$ each. Microglia purified from hypothalami of AF animals did not show differences in basal inflammatory genes (IL-6 and TNF- $\alpha)$ expression $(\mathbf{k}$, I) $(n=5-6 /$ treatment group). However, microglia from hypothalami of AF animals challenged with LPS showed an increase in IL-6 (k) ( $n=5-7 / g r o u p)$ and TNF- $\alpha$ (I) ( $n=6-15 /$ group) in comparison with control animals. Co-administration of minocycline with alcohol (AF $+M$ ) diminished the exaggerated IL- 6 (k) and TNF- $\alpha$ (I) response to LPS in AF animals. Data are mean \pm SEM and were analyzed using two-way ANOVA followed by Bonferroni post-test $(\mathbf{a}, \mathbf{b})$ or analyzed using one-way ANOVA followed by Newman-Keuls post-tests. ${ }^{*} p<0.05$; ${ }^{* *} p<0.01$; and ${ }^{* * *} p<$ 0.001 significantly different between groups as indicated by a bar on the top of the graph

\section{RESULTS}

Neonatal alcohol activates pro-inflammatory cytokine expression in hypothalamic microglia

In these studies, we sought to determine if PND 2-6 alcohol exposure $(2.5 \mathrm{~g} / \mathrm{kg})$ altered microglia signature marker $\mathrm{Cd} 11 \mathrm{~b}$ in hypothalamus and pro-inflammatory cytokine gene expression from microglia isolated from PND 6 hypothalamus (see Fig. 1a for experimental paradigm). Five days of alcohol exposure increased microglial $\mathrm{Cd} 11 \mathrm{~b}+$ expression in the PVN (treatment, $F(2,36)=$ $46.29, p<0.0001$; time, $F(2,36)=8.26, p<0.0007$ ) (Fig. 1b) and ARC (treatment, $F(2,36)=15.69, p<0.0001$; time, $F(2,36)=10.68, p<$ 0.0002 ) (Fig. 1c) of the hypothalamus of AF animals compared to control animals. In addition, hypothalamic microglia from PND 6 AF animals showed increased mRNA levels of interleukin-6 (IL-6) ( $F$ $(2,13)=6.988, p<0.01)$ (Fig. 1g), tumor necrosis factor a (TNF-a) $(F$ $(2,12)=7.109, p<0.01)$ (Fig. 1h), colony stimulating factor 1 receptor (CSF-1R) $(F(2,12)=37.27, p<0.0001)$ (Fig. 1i), and toll-like receptor 4 (TLR-4) $(H=7.081,2$ d.f., $p<0.05)$ (Fig. 1j) compared to microglia from $A D$ and PF animals. mRNA levels of interleukin 1beta (IL1 $\beta)(F(2,14)=0.526, p>0.05)$ and monocyte chemoattractant protein-1 (MCP-1/CCL2) $(F(2,15)=0.190, p>0.05)$ were not significantly different between $\mathrm{AF}$ microglia and control microglia (data not shown). These results are in agreement with the previous findings that early life exposure to alcohol results in changes in brain region-specific increases in inflammatory cytokines [16, 35-37], and further suggest that early life exposure to alcohol during PND 2-6 activates microglia and increases microglial pro-inflammatory gene expression in hypothalamus. Similarly, studies utilizing cultured microglia derived from hypothalamus of neonatal rat pups, showed exposure to a high dose of alcohol ( $50 \mathrm{mM} \mathrm{ETOH}$ ) for $24 \mathrm{~h}$ resulted in increased gene expression of TNF-a $(t(9)=9.146, p<0.0001)$ (Fig S1b), CSF-1R $(t$ $(8)=2.496, p<0.05)(\mathrm{S} 1 \mathrm{c})$, and TLR-4 $(t(5)=1.985, p<0.05)(\mathrm{S} 1 \mathrm{~d})$, while expression of IL-6 in ETOH-exposed cultured microglia was not significantly changed $(t(10)=0.691, p>0.05)(\mathrm{S} 1 \mathrm{a})$.

Minocycline attenuates long-term immune potentiation following neonatal alcohol exposure

In addition to the immediate effects, our studies aimed to investigate whether early life alcohol has long-term effects on the HPA axis and hypothalamic microglia, and whether these effects might be normalized by blocking microglia activation with minocycline treatment at the time of alcohol exposure. To this end (Fig. 2), we measured stress hormones and microglia activation and inflammation at baseline and $2 \mathrm{~h}$ after LPS challenge in adult $A F, A F+M, P F$, and $A D$ animals. $A F$ adult animals did not significantly differ in basal plasma stress hormone levels, but showed significantly increased CORT (treatment, $F$ $(5,49)=3.33, p=0.011$; time, $F(1,49)=198.24, p<0.0001)$ (Fig. 2a) and ACTH (treatment, $F(5,47)=4.42, p=0.0022$; time, $F(1,47)=$
122.32, $p<0.0001$ ) (Fig. 2b) response to the LPS challenge. The hormonal hyper-response to LPS was normalized by minocycline treatment $(A F+M)$ during the PND 2-6 alcohol exposure time (Fig. 2a, b).

Microglial number and activation levels were measured by immunohistochemical detection of IBA- $1+$ and $\mathrm{Cd} 1 \mathrm{1b}+$ cell count, respectively, in the ARC and PVN regions of the hypothalamus in the adult rats. Under basal condition activated microglial number did not differ between treatment groups in the ARC region of the hypothalamus, but AF animals showed increased microglial number in response to LPS challenge that was prevented by minocycline treatment $(F(6,28)=4.283, p=$ 0.003 ) (Fig. 2c). Total microglial number in the ARC also did not differ between treatment groups, but AF animals showed increased microglial number in response to LPS compared to PF and $\mathrm{AF}+\mathrm{M}$ treated animals $(F(6,28)=8.779, p=0.0001)$ (Fig. $2 \mathrm{~d}$ ). Adult AF animals showed increased activated microglial number basally and $2 \mathrm{~h}$ after LPS challenge in the PVN region compared to control animals $(F(6,28)=18.08, p<0.0001)$ (Fig. 2e). AF animals also showed increased total microglial cell count basally and after LPS challenge in the PVN $(F(6,28)=5.102, p=0.0012)$ (Fig. 2f). Minocycline treatment normalized the increased total microglial number in the PVN in AF animals.

Finally, mRNA levels of pro-inflammatory factors previously found to be acutely elevated in microglia after PND 2-6 alcohol exposure were measured in adult animals basally and $2 \mathrm{~h}$ post LPS. Basal levels of microglial IL-6 (Fig. 2k) and TNF-a (Fig. 2l) were not significantly different between $A D, P F$, and $A F$, but $A F$ animals showed increased IL-6 $(F(6,32)=14.39, p<0.0001)$ and TNF-a $(H=15.45,6$ d.f., $p<0.05)$ gene expression after LPS challenge compared to control animals, an effect which was again normalized by minocycline treatment (Fig. 2k, I). Neither CSF-1R $(F(5,31)$ $=0.173, p>0.05)$ (Fig S2a) nor TLR-4 $(F(5,38)=1.060, p>0.05)$ (Fig S2b) microglial gene expression were significantly altered in AF animals at baseline or after LPS. Together, these results show that early life alcohol exposure results in long-term hormonal and microglial hyper-response to immune stress, an effect which can be prevented by microglial inhibition by minocycline at the time of alcohol exposure.

Neonatal alcohol's effects on epigenetic mediators in hypothalamic microglia

As our studies showed adult microglial hyper-response produced by neonatal alcohol exposure, we focused our attention on investigating a possible epigenetic mechanism for the immediate and long-term effects of neonatal alcohol on microglial activation and later-life stress hypersensitivity. Thus, we first investigated whether PND 2-6 in vivo alcohol exposure affected a variety of epigenetic mediators of inflammatory gene expression in hypothalamic microglia. Microglia from AF pups showed 

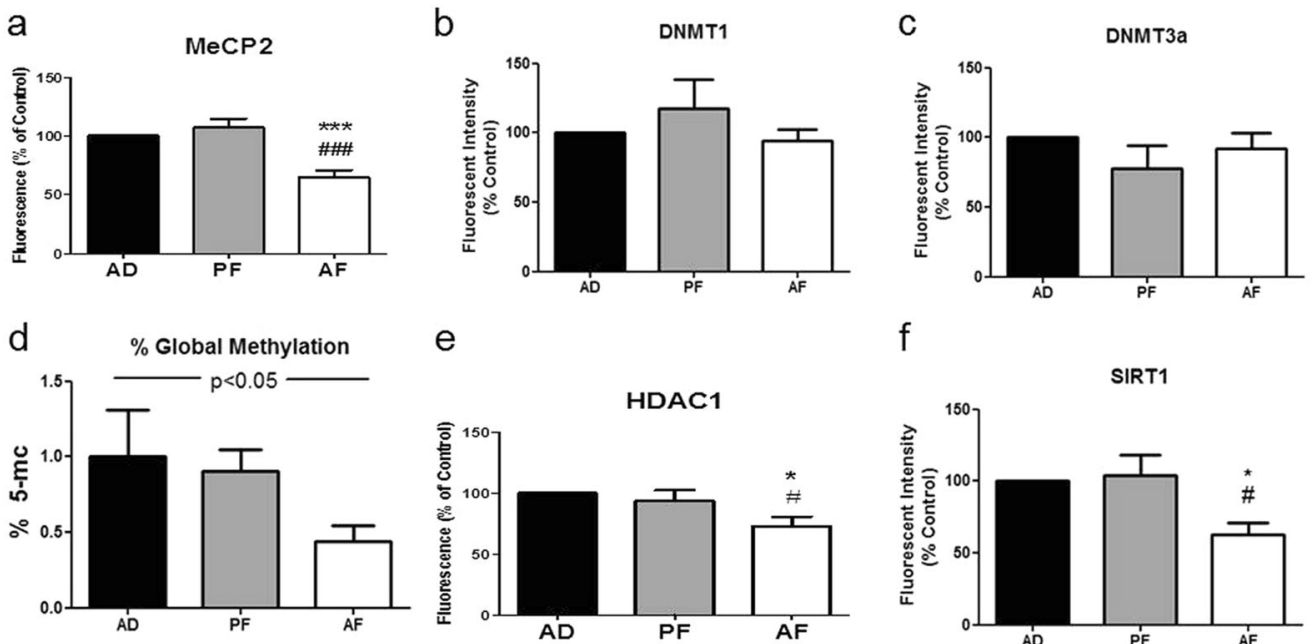

f
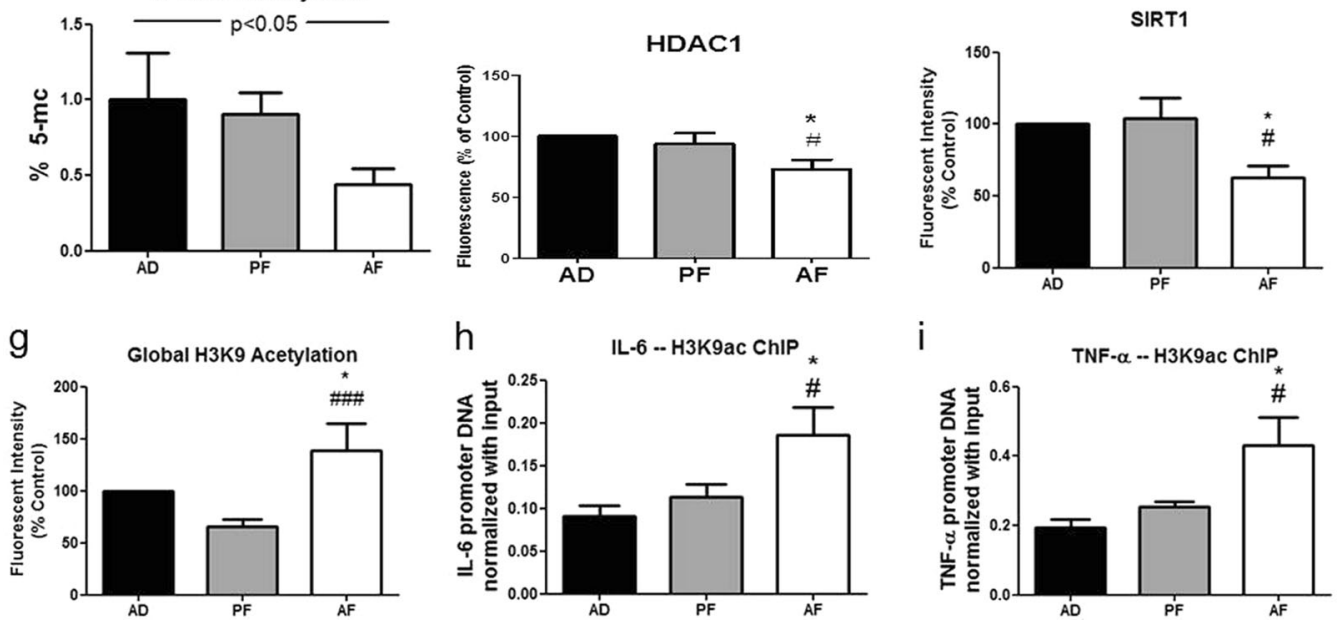

Fig. 3 Neonatal alcohol effects on epigenetic mediators in hypothalamic microglia in vivo. Hypothalamic microglia from PND 6 AD, PF, and AF rat pups were measured for protein levels of epigenetic mediators of inflammatory gene transcription. In vivo, hypothalamic microglia from AF animals showed decreased levels of negative regulators of transcription including methyl CpG-binding protein 2 (MeCP2) (a) ( $n=5-6 /$ treatment group) and decreased global DNA methylation (d) $(n=4-6)$ compared to control animals. Levels of DNMT1 (b) ( $n=7-9)$ and DNMT3a (c) ( $n=4-11)$ were not significantly different in microglia from AF animals compared to AD and PF controls. In addition, compared to microglia from control pups, microglia from AF pups showed decreased protein levels of histone deacetylases HDAC1 (e) $(n=9-11)$ and sirtuin 1 (SIRT1) (f) $(n=8)$, while showing increased global acetylation of their target histone H3 lysine 9 (H3K9ac) (g) ( $n=6-12)$, a positive regulator of inflammatory gene transcription. Finally, ChIP of H3K9ac from hypothalamic microglia showed increased enrichment of the IL- 6 (h) and TNF- $\alpha$ (i) promoter gene regions in AF pups compared to PF and AD pups $(n=4)$. Data are mean \pm SEM $(n=4-11)$, and were analyzed

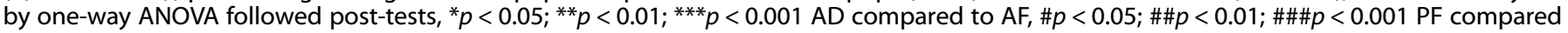
to $A F$

significantly decreased levels of negative regulators of transcription, including decreased protein levels of methyl-CpG-binding protein $2(F(2,14)=18.65, p<0.0001)(\mathrm{MeCP} 2)$ (Fig. 3a), HDAC1 $(F$ $(2,27)=4.830, p<0.05)$ (Fig. 3e), and SIRT1 $(F(2,21)=5.706, p<$ $0.05)$ (Fig. 3f), and decreased global DNA methylation $(F(2,13)=$ 3.830, $p<0.05$ ) (Fig. 3d) compared to control pups. Protein levels of DNA methyltransferases DNMT1 $(F(2,21)=1.045, p>0.05)$ (Fig. 3b) and DNMT3a $(F(2,21)=0.8783, p>0.05)$ (Fig. 3c) were not significantly altered in AF hypothalamic microglia compared to control microglia. In addition, global acetylation of histone 3 lysine 9 (H3K9ac) $(H=15.57,2$ d.f., $p<0.001)$ (Fig. 3g), a target of HDAC1 and SIRT1, was increased in hypothalamic microglia from AF animals compared to PF and $A D$ animals. To examine if the increased $\mathrm{H} 3 \mathrm{~K} 9 \mathrm{ac}$ was related to the increased gene expression of pro-inflammatory cytokines in microglia observed after PND 2-6 alcohol treatment, we performed ChIP of H3K9ac in PND 6 AD, PF, and $\mathrm{AF}$ hypothalamic microglia. We found hypothalamic microglia from AF pups showed increased H3K9ac enrichment of IL-6 $(F(2,9)$ $=4.926, p<0.05)$ (Fig. 3h) and TNF-a $(F(2,9)=6.365, p<0.05)$ (Fig. 3i) gene promoter regions compared to hypothalamic microglia from $A D$ and PF pups. These studies show decreased levels of HDACs, increased H3K9ac, and increased specific H3K9ac enrichment at the IL- 6 and TNF- $a$ gene promoters in PND 2-6 alcohol-exposed microglia correlate with increased gene expression of these pro-inflammatory cytokines, and demonstrate a likely epigenetic mechanism for developmental alcohol activation of hypothalamic microglia. In vitro results largely concurred with our in vivo data showing developmental alcohol exposure decreases
HDACs, increases global H3K9 acetylation, and increases H3K9aC enrichment at specific pro-inflammatory gene promoters in cultured hypothalamic microglia (see Fig. S3).

Neonatal alcohol has long-term effects on hypothalamic microglial epigenetic mediators in vivo

We next investigated whether epigenetic changes in hypothalamic microglia induced by early life alcohol persist into adulthood as a possible mechanism for microglial programming by alcohol. Microglia from AF adult animals pre-exposed to neonatal alcohol showed several enduring epigenetic changes. Adult AF microglia showed basally decreased levels of SIRT1 $(F(5,28)=6.165, p<$ 0.001 ) (Fig. 4c) compared to microglia from control animals. Kruskal-Wallis test of DNMT1 levels in adult microglia approached significance $(H=10.68,5$ d.f., $p=0.058)$, and post-tests revealed basal DNMT1 levels were decreased in AF microglia compared to controls (Fig. 4e). Post LPS, levels of SIRT1 and DNMT1 did not significantly differ between treatment groups. ANOVA for DNMT3a levels in adult microglia reached significance $(F(5,28)=2.774, p<$ $0.05)$, but post-tests did not reveal significant differences between AF microglia and controls $(p>0.05)$. Thus, levels of DNMTs showed a statistical trend for decreasing in $\mathrm{AF}$ microglia compared to control groups. In addition to baseline differences, adult AF microglia also differed in response to LPS. Post LPS, adult AF microglia showed significantly decreased HDAC1 levels $(F(5,16)=$ $15.98, p<0.0001$ ) (Fig. 4a), and increased global acetylation of HDAC1's target H3K9ac $(H=12.85,5$ d.f., $p<0.05)$, compared to $A D$ controls (Fig. 4b). To connect these epigenetic modifications 

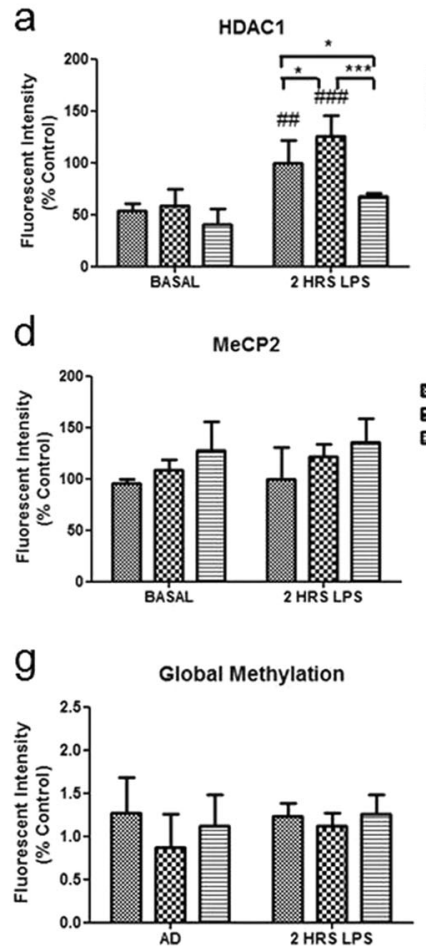

b
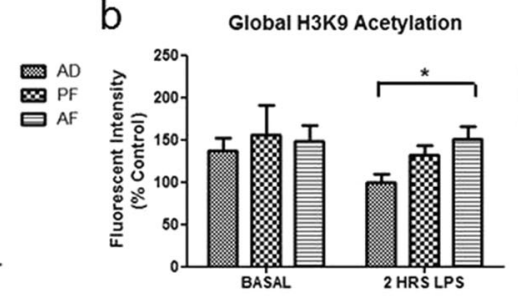

e
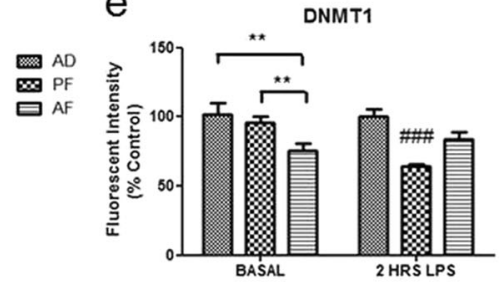

$\mathrm{h}$

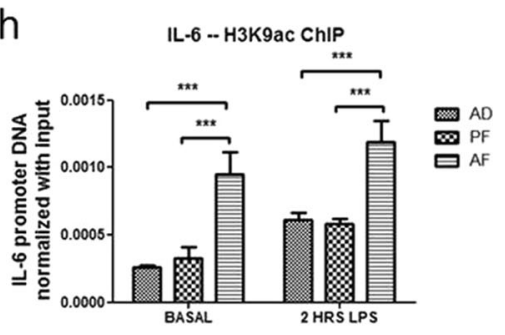

C

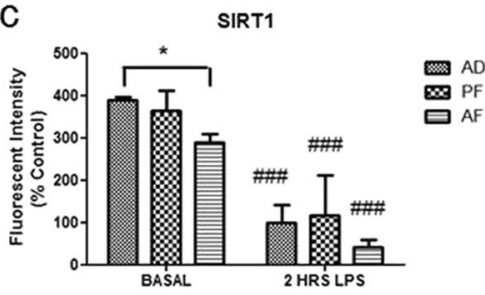

f

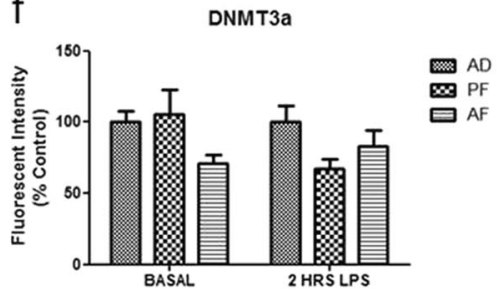

i

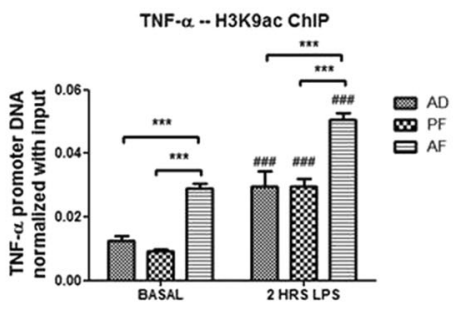

Fig. 4 Neonatal alcohol has long-term programming effects on hypothalamic microglial epigenetic mediators in vivo. The long-term effects of neonatal (PND 2-6) alcohol exposure on protein levels of epigenetic mediators in hypothalamic microglia of adult (PND 90) rats were examined at baseline (basal) and $2 \mathrm{~h}$ after an immune challenge (LPS, $0.1 \mathrm{mg} / \mathrm{kg}$ ). Basal levels of SIRT1 (c) in hypothalamic microglia from AF adults were significantly decreased. Levels of DNMT1 (e) and DNMT3a (f) in AF adults showed statistical trends for decreasing compared to control groups. Neonatal exposure to alcohol also showed altered microglial epigenetic response to an immune challenge in the adult. Two hours after LPS exposure, AF adults showed decreased HDAC1 protein compared to control animals (a), and also showed increased global H3K9 acetylation (b) as compared to controls. SIRT1 (c) levels in microglia were decreased by LPS, but did not significantly differ between AF and control animals. Adult AF animals did not significantly differ in microglial levels of MeCP2 (d), DNMT3a (f) or global DNA methylation (g) compared to control animals. Finally, ChIP of H3K9ac from hypothalamic microglia showed increased enrichment of the IL- 6 (h) and TNF- $\alpha$ (i) promoter gene regions in AF adult animals compared to PF and AD controls at baseline and $2 \mathrm{~h}$ post LPS. Data are mean \pm SEM, and were

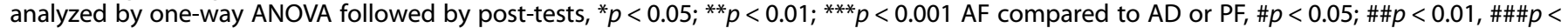
0.001 basal compared to $2 \mathrm{~h}$ post LPS. For all protein measurements and the methylation assay, sample sizes were $n=4-8 /$ treatment group, and for ChIP experiments, sample sizes were $n=6-8$

with changes observed in gene expression (Fig. 2), we again utilized ChIP of H3K9ac in hypothalamic microglia from AF, PF, and $A D$ adults. We found an enduring increase in H3K9ac enrichment at the IL-6 $(F(5,36)=12.44, p<0.0001)$ (Fig. 4h) and TNF-a $(F(5,40)$ $=31.85, p<0.0001$ ) (Fig. 4i) promoter gene regions in hypothalamic microglia from AF animals both at baseline and $2 \mathrm{~h}$ after LPS. These studies show long-term epigenetic changes (decreases in HDACs and increased H3K9ac of pro-inflammatory genes) both at baseline and after an immune challenge in adult hypothalamic microglia following early life alcohol exposure.

\section{DISCUSSION}

Recently, a number of studies in adult and adolescent animals have found the effects of binge-like alcohol on neuroinflammation and microglia to additional alcohol doses, LPS, or stress to be additive, causing a hyper-response to subsequent immune challenges [38-40]. However, it is unknown whether the effects of early life alcohol on the neuroimmune system and microglia are long-lasting. Thus, our studies utilizing PND 2-6 alcohol exposed rat pups, investigated the immediate and long-term effects of neonatal alcohol on microglia in the hypothalamus. We observed alcohol exposure during early development acutely activated microglial cells and microglial-specific expression of proinflammatory factors in hypothalamus after the alcohol exposure (Fig. 1, S1). Importantly, the effects of neonatal alcohol on hypothalamic microglia persisted into adulthood (PND 90). Interestingly, more microglia were present (Fig. 2C) and were reactive (Fig. 2e) in the PVN basally in adults pre-exposed to developmental alcohol, indicating some long-term changes in basal activity of PVN microglia as a result of neonatal alcohol. While basal measurements of most other parameters measured were not altered, hypothalamic microglial response to LPS challenge-both activation and pro-inflammatory gene expression-was significantly increased in AF animals (Fig. 2), indicating a priming effect of developmental alcohol on hypothalamic microglia, reminiscent of microglial priming effects observed after stress or injury [22-24].

Alongside priming of microglia to an immune challenge, stress axis response to LPS in AF animals was hyper-sensitized, indicated by exaggerated plasma ACTH and CORT response (Fig. 2a,b). Interestingly, a recent study by Walter et al. [40], also found a correlation between increased microglial activation and peripheral CORT response as a consequence of chronic alcohol in a rat model. The mechanism for the relationship between alcoholinduced microglial activation and systemic HPA axis disruption are currently under study in our lab, and evidence suggests microgliainduced neurotoxicity of BEP neurons by developmental alcohol is a major factor $[15,16]$, but more research is clearly warranted in this area. Importantly, the hyper-sensitized microglia and stress axis response to LPS were diminished by simultaneous treatment with minocycline at the time of alcohol exposure. Though 


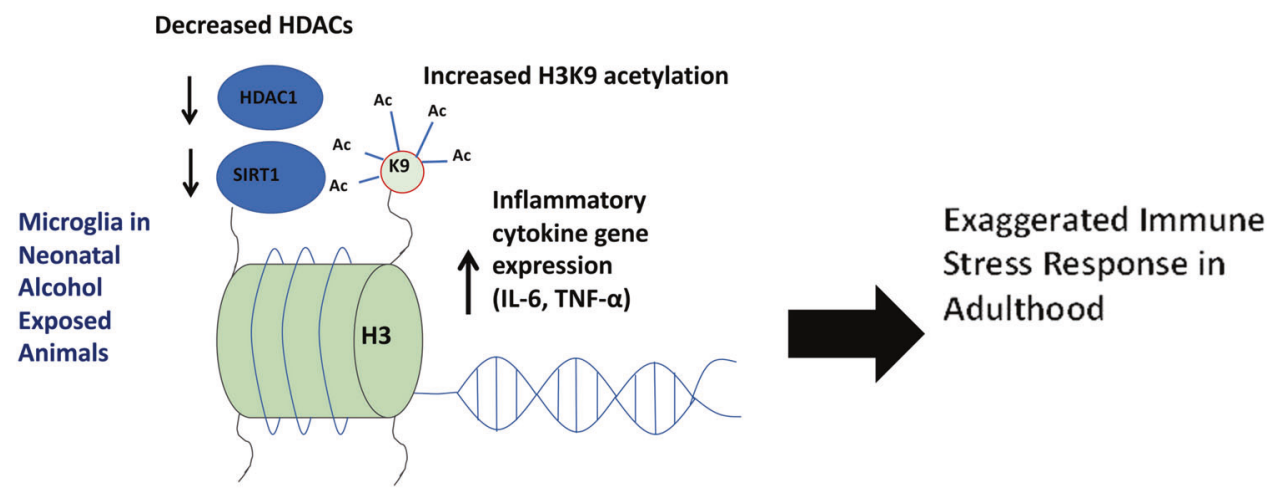

Fig. 5 Theoretical mechanism for activation and priming of microglia by neonatal alcohol. Exposure to alcohol during the neonatal time period results in acute and long-term increases in inflammatory gene expression (IL- 6 and TNF- $\alpha$ ) alongside decreases in negative regulators of inflammatory gene transcription, such as HDAC1 and SIRT1 and increased global acetylation of their target histone 3 lysine 9 (H3K9ac). In addition, neonatal alcohol exposure increases the association between H3K9ac and the promoter regions of IL- 6 and TNF- $\alpha$ both at the time of alcohol exposure (PND6) and later in life during adulthood, both at baseline and post LPS. These results demonstrate an epigenetic mechanism for early life alcohol exposure's acute activation and long-term priming of microglia sensitivity to immune stress

minocycline is not a selective microglial inhibitor [41], it has been shown to significantly block M1 microglial activation [42]. Minocycline treatment has been shown to decrease fetal alcohol-induced increase in microglial activation and neurotoxicity [16] and neuroinflammation and alcohol drinking in adult rodent models $[43,44]$, although the mechanism for these neuroprotective effects is unknown, and requires further study. Together, these studies suggest minocycline may have therapeutic potential for the treatment of alcohol-related disorders, such as FASDs. In addition, our results suggest that microglia activation during the time of alcohol exposure is necessary for the later-life hypersensitivities observed in hypothalamic microglia and hormonal stress axis response, directly correlating early life microglial activation with later life immune and stress axis disruption.

Given these results, we asked what is the mechanism for the long-term priming of microglia by developmental alcohol? Inflammatory response-both the initiation of inflammation after injury or illness and the inhibition of excessive inflammation-is tightly regulated by epigenetic mechanisms [26]. In addition, alcohol exposure has been shown to induce epigenetic changes in somatic cells, especially in brain, including changes in DNA methylation $[45,46]$, methylated DNA-binding proteins, such as MeCP2 [47, 48], HDACs [49], and histone modifications [50, 51], although few studies have focused on the epigenetic effects of $\mathrm{ETOH}$ on specific cell types. No studies to our knowledge have investigated the effects of developmental alcohol on epigenetic mediators in microglia to date. Thus, we examined the immediate and long-lasting effects of developmental alcohol exposure on hypothalamic microglial cells. Neonatal alcohol exposure decreased negative regulators of transcription including global DNA methylation (Fig. 3d) and MeCP2, which binds methylated DNA (Fig. 3a). In addition to decreased methylation and MeCP2, PND 2-6 alcohol produced an immediate decrease in hypothalamic microglial histone deacetylases HDAC1 and SIRT1 (Fig. 3e, f), increased global acetylation of their target H3K9 (Fig. 3g), and increased specific H3K9ac enrichment at the IL- 6 and TNF-a genes (Fig. 3h, i). Our results are comparable to studies in peripheral immune cells, which show both increased acetylation of H3K9 and decreases in HDAC association with promoters are critical for the activation of the transcriptional immune response [52, 53]. Thus, our results demonstrate a likely epigenetic mechanism by which developmental alcohol activates pro-inflammatory gene expression in hypothalamic microglia at the time of alcohol exposure.

Significantly, we found that PND 2-6 alcohol-induced decreases in HDACs, increased H3K9 acetylation, and increased H3K9ac enrichment at IL- 6 and TNF-a gene promoters persisted through adulthood in hypothalamic microglia. Thus, H3K9ac enrichment at IL- 6 and TNF-a promoters after early life alcohol exposure may serve as a marker for cellular memory of the immune event, thus pre-disposing hypothalamic microglia to a hyper-inflammatory response to later life immune challenges. This demonstrates a potential epigenetic mechanism for microglial priming by developmental alcohol (Fig. 5). Histone modifications, such as acetylation, are dynamic, and can be turned on or off to adjust transcription as needed by the cell. However, some histone modifications, such as $\mathrm{H} 3 \mathrm{~K} 9 \mathrm{ac}$, may be relatively stable over time and even across mitotic cell divisions, thus preserving epigenetic changes as a cellular memory [31]. In the peripheral immune system, H3K9 hyper-acetylation at certain genes was found to be a key factor in the rapid immune response of memory CD8+ T-cells compared to naïve T-cell precursors [54]. A similar mechanism might be at play in alcohol-primed microglia. Another possibility is that the H3K9ac marker at IL- 6 and TNF-a promoters was preserved due to a long-term decrease in HDACs, as we observed HDAC1 and SIRT1 remained decreased in hypothalamic microglia of adult AF animals (Fig. 4a, c). Similarly, long-term decreases in HDAC activity are also observed in peripheral alveolar immune cells in chronic obstructive pulmonary disease (COPD), a pathology involving excessive chronic inflammation in lungs [55]. While our results showed lasting changes in H3K9ac and HDACs in hypothalamic microglia, which were correlated with microglial immune hypersensitivity, we did not observe persistent changes in global DNA methylation (Fig. 4g) or MeCP2 (Fig. 4d), although we did observe a long-lasting decrease in basal DNMT1 levels in AF hypothalamic microglia (Fig. 4e). This change may suggest the possibility of additional long-lasting epigenetic marks at the level of DNA methylation on specific microglial immune response genes produced by developmental alcohol, a possibility which should be addressed by future studies. Indeed, epigenetic changes in histone modifications and DNA methylation occur in consort [56], so future studies might evaluate the long-lasting effect of developmental alcohol on microglial function at multiple epigenetic levels using high-throughput methods like ChIP sequencing or DNA methylome sequencing.

In summary, PND 2-6 alcohol exposure primes hypothalamic microglia and HPA axis response to an adult immune challenge, and this priming effect can be blocked by minocycline treatment during the time of alcohol exposure. While our experiments focused on stress axis and microglia function, follow-up studies are necessary to assess additional functional and behavioral consequences of developmental alcohol exposure, and to evaluate whether blocking microglia activation ameliorates these 
effects. Future studies should further evaluate minocycline as a potential pharmacotherapy for FASDs and other alcohol-related disorders. In addition, follow-up studies might utilize microgliaspecific gene targeting methods to inhibit microglia or to target specific gene expression of epigenetic mediators, such as HDACs in microglia. Concomitant long-lasting epigenetic changes in HDACs and H3K9ac in adult hypothalamic microglia from animals pre-exposed to neonatal alcohol suggest a possible epigenetic mechanism for microglial priming by developmental alcohol (Fig. 5). Future studies should build on these results by obtaining a comprehensive picture of the shifting epigenetic landscape involved in microglial priming by developmental alcohol.

\section{FUNDING AND DISCLOSURE}

This work is partly supported by NIH grants R37AA08757 to DKS, and F32AA023434 grant to LC, for this research under the R37AA08757-12-14S1 to TF and R37AA08757-19-22S1 to MC

\section{ACKNOWLEDGEMENTS}

We thank Gayathri Narayanan and Rahul Nayar for their assistance in gene expression studies. We thank Gregory Berger for his technical assistance in animal experiments.

\section{ADDITIONAL INFORMATION}

Supplementary Information accompanies this paper at (https://doi.org/10.1038/ s41386-019-0326-7)

Competing interests: The authors declare no competing interests.

Publisher's note: Springer Nature remains neutral with regard to jurisdictional claims in published maps and institutional affiliations.

\section{REFERENCES}

1. May PA, et al. Prevalence of fetal alcohol spectrum disorders in 4 US communities. JAMA. 2018;319:474-82.

2. Lange S, Probst C, Gmel G, Rehm J, Burd L, Popova S. Global prevalence of fetal alcohol spectrum disorder among children and youth: a systematic review and meta-analysis. JAMA Pediatr. 2017;171:948-56.

3. Riley EP, Infante MA, Warren KR. Fetal alcohol spectrum disorders: an overview. Neuropsychol Rev. 2011;21:73-80.

4. Caputo C, Wood E, Jabbour L. Impact of fetal alcohol exposure on body systems: a systematic review. Birth Defects Res C. 2016;108:174-80.

5. Moore EM, Riley EP. What happens when children with fetal alcohol spectrum disorders become adults? Curr Dev Disord Rep. 2015;2:219-27.

6. Zhang $\mathrm{X}$, Sliwowska JH, Weinberg J. Prenatal alcohol exposure and fetal programming: effects on neuroendocrine and immune function. Exp Biol Med. 2005;230:376-88.

7. Mead EA, Sarkar DK. Fetal alcohol spectrum disorders and their transmission through genetic and epigenetic mechanisms. Front Genet. 2014;5:154.

8. Johnson S, Knight R, Marmer DJ, Steele RW. Immune deficiency in fetal alcohol syndrome. Pediatr Res. 1981;15:908-11.

9. Olah M, Biber K, Vinet J, Boddeke HW. Microglia phenotype diversity. CNS Neurol Disord Drug Targets. 2011;10:108-18.

10. Chastain LG, Sarkar DK. Role of microglia in regulation of ethanol neurotoxic action. Int Rev Neurobiol. 2014;118:81-103.

11. Crews FT, Sarkar DK, Qin L, Zou J, Boyadjieva N, Vetreno RP. Neuroimmune function and the consequences of alcohol exposure. Alcohol Res. 2015;37:331-41. 344-51

12. He J, Crews FT. Increased MCP-1 and microglia in various regions of the human alcoholic brain. Exp Neurol. 2008;210:349-58.

13. Alfonso-Loeches S, Pascual M, Guerri C. Gender differences in alcohol-induced neurotoxicity and brain damage. Toxicology. 2013;311:27-34.

14. Wong EL, Stowell RD, Majewska AK. What the spectrum of microglial functions can teach us about fetal alcohol spectrum disorder. Front Synaptic Neurosci. 2017;9:11.

15. Boyadjieva NI, Sarkar DK. Role of microglia in ethanol's apoptotic action on hypothalamic neuronal cells in primary cultures. Alcohol Clin Exp Res. 2010;34:1835-42.

16. Shrivastava $P$, et al. Mu-opioid receptor and delta-opioid receptor differentially regulate microglial inflammatory response to control proopiomelanocortin neuronalapoptosis in the hypothalamus: effects of neonatal alcohol. J Neuroinflamm. 2017;14:83.

17. Bodnar TS, Hill LA, Weinberg J. Evidence for an immune signature of prenatalalcohol exposure in female rats. Brain Behav Immun. 2016;58:130-41.

18. Topper LA, Baculis BC, Valenzuela CF. Exposure of neonatal rats to alcohol has differential effects on neuroinflammation and neuronal survival in the cerebellum and hippocampus. J Neuroinflamm. 2015;12:160.

19. Tiwari V, Chopra K. Resveratrol abrogates alcohol-induced cognitive deficits by attenuating oxidative-nitrosative stress and inflammatory cascade in the adult rat brain. Neurochem Int. 2013;62:861-9.

20. McClain JA, et al. Adolescent binge alcohol exposure induces long-lasting partial activation of microglia. Brain Behav Immun. 2011;25(Suppl 1):S120-8.

21. Vetreno RP, Lawrimore CJ, Rowsey PJ, Crews FT. Persistent adult neuroimmune activation and loss of hippocampal neurogenesis following adolescent ethanol exposure: blockade by exercise and the anti-inflammatory drug indomethacin. Front Neurosci. 2018;12:200.

22. Williamson LL, Sholar PW, Mistry RS, Smith SH, Bilbo SD. Microglia and memory: modulation by early-life infection. J Neurosci. 2011;31:15511-21.

23. Fenn AM, Gensel JC, Huang Y, Popovich PG, Lifshitz J, Godbout JP. Immune activation promotes depression 1 month after diffuse brain injury: a role for primed microglia. Biol Psychiatry. 2014;76:575-84.

24. Niraula A, Sheridan JF, Godbout JP. Microglia priming with aging and stress. Neuropsychopharmacology. 2017;42:318-33.

25. Perry VH, Holmes C. Microglial priming in neurodegenerative disease. Nat Rev Neurol. 2014;10:217-24.

26. Kaminska B, Mota M, Pizzi M. Signal transduction and epigenetic mechanisms in the control of microglia activation during neuroinflammation. Biochim Biophys Acta. 2016;1862:339-51.

27. Chater-Diehl EJ, Laufer BI, Singh SM. Changes to histone modifications following prenatal alcohol exposure: an emerging picture. Alcohol. 2017;60:41-52.

28. Laufer BI, Chater-Diehl EJ, Kapalanga J, Singh SM. Long-term alterations to DNA methylation as a biomarker of prenatal alcohol exposure: from mouse models to human children with fetal alcohol spectrum disorders. Alcohol. 2017;60:67-75.

29. Hellemans KG, et al. Prenatal alcohol exposure: fetal programming and later life vulnerability to stress, depression and anxiety disorders. Neurosci Biobehav Rev. 2010;34:791-807.

30. Weinberg J, et al. Prenatal alcohol exposure: foetal programming, the hypothalamic-pituitary-adrenal axis and sex differences in outcome. J Neuroendocrinol. 2008;20:470-88.

31. Bilbo SD, Frank A. Beach award: programming of neuroendocrine function by early-life experience: a critical role for the immune system. Horm Behav 2013;63:684-91.

32. Klintsova AY, Hamilton GF, Boschen KE. Long-term consequences of developmental alcohol exposure on brain structure and function: therapeutic benefits of physical activity. Brain Sci. 2012;3:1-38.

33. Paxinos G, Franklin KBJ. The mouse brain in stereotaxic coordinates. San Diego, CA: Academic; 2001.

34. Ramachandra R, Subramanian T. Atlas of the neonatal rat brain. Boca Raton, FL: CRC Press; 2011.

35. Kane $\mathrm{CJ}$, et al. Protection of neurons and microglia against ethanol in a mouse model of fetal alcohol spectrum disorders by peroxisome proliferator-activated receptor- $\gamma$ agonists. Brain Behav Immun. 2011;25(Suppl 1):S137-45.

36. Ahlers KE, Karaçay B, Fuller L, Bonthius DJ, Dailey ME. Transient activation of microglia following acute alcohol exposure in developing mouse neocortex is primarily driven by BAX-dependent neurodegeneration. Glia. 2015;63: 1694-713.

37. Boschen KE, Ruggiero MJ, Klintsova AY. Neonatal binge alcohol exposure increases microglial activation in the developing rat hippocampus. Neuroscience. 2016;324:355-66.

38. Marshall SA, Geil CR, Nixon K. Prior binge ethanol exposure potentiates the microglial response in a model of alcohol-induced neurodegeneration. Brain Sci. 2016;6:pii: E16

39. Qin L, He J, Hanes RN, Pluzarev O, Hong JS, Crews FT. Increased systemic and brain cytokine production and neuroinflammation by endotoxin following ethanol treatment. J Neuroinflamm. 2008;5:10.

40. Walter TJ, Vetreno RP, Crews FT. Alcohol and stress activation of microglia and neurons: brain regional effects. Alcohol Clin Exp Res. 2017;41:2066-81.

41. Möller T, et al. Critical data-based re-evaluation of minocycline as a putative specific microglia inhibitor. Glia. 2016;64:1788-94.

42. Kobayashi K, et al. Minocycline selectively inhibits M1 polarization of microglia. Cell Death Dis. 2013;4:e525.

43. Qin L, Crews FT. Chronic ethanol increases systemic TLR3 agonist-induced neuroinflammation and neurodegeneration. J Neuroinflamm. 2012;9:130.

44. Agrawal RG, Hewetson A, George CM, Syapin PJ, Bergeson SE. Minocycline reduces ethanol drinking. Brain Behav Immun. 2011:25(Suppl 1):S165-9. 
Early life alcohol exposure primes hypothalamic microglia to later-life...

LG Chastain et al.

1588

45. Garro AJ, McBeth DL, Lima V, Lieber CS. Ethanol consumption inhibits fetal DNA methylation in mice: implications for the fetal alcohol syndrome. Alcohol Clin Exp Res. 1991;15:395-8.

46. Chen Y, Ozturk NC, Zhou FC. DNA methylation program in developing hippocampus and its alteration by alcohol. PLoS One. 2013;8:e60503.

47. Kim $P$, et al. Effects of ethanol exposure during early pregnancy in hyperactive, inattentive and impulsive behaviors and MeCP2 expression in rodent offspring. Neurochem Res. 2013;38:620-31.

48. Gangisetty O, Bekdash R, Maglakelidze G, Sarkar DK. Fetal alcohol exposure alters proopiomelanocortin gene expression and hypothalamic-pituitary-adrenal axis function via increasing MeCP2 expression in the hypothalamus. PLoS One. 2014;9:e113228.

49. Moonat S, Sakharkar AJ, Zhang H, Tang L, Pandey SC. Aberrant histone deacetylase2-mediated histone modifications and synaptic plasticity in the amygdala predisposes to anxiety and alcoholism. Biol Psychiatry. 2013;73:763-73.

50. Sakharkar AJ, et al. Effects of histone deacetylase inhibitors on amygdaloid histone acetylation and neuropeptide $\mathrm{Y}$ expression: a role in anxiety-like and alcohol-drinking behaviours. Int J Neuropsychopharmacol. 2014;17:1207-20.
51. Veazey KJ, Parnell SE, Miranda RC, Golding MC. Dose-dependent alcohol-induced alterations in chromatin structure persist beyond the window of exposure and correlate with fetal alcohol syndrome birth defects. Epigenetics Chromatin. 2015;8:39.

52. Rybtsova N, Leimgruber E, Seguin-Estévez Q, Dunand-Sauthier I, Krawczyk M, Reith W. Transcription-coupled deposition of histone modifications during MHC class II gene activation. Nucleic Acids Res. 2007;35:3431-41.

53. Falvo JV, Jasenosky LD, Kruidenier L, Goldfeld AE. Epigenetic control of cytokine gene expression: regulation of the TNF/LT locus and T helper cell differentiation. Adv Immunol. 2013;118:37-128.

54. Fann $\mathrm{M}$, et al. Histone acetylation is associated with differential gene expression in the rapid and robust memory $\mathrm{CD} 8(+)$ T-cell response. Blood. 2006;108:3363-70.

55. Ito $\mathrm{K}$, et al. Decreased histone deacetylase activity in chronic obstructive pulmonary disease. N Engl J Med. 2005:352:1967-76.

56. Cedar H, Bergman Y. Linking DNA methylation and histone modification: patterns and paradigms. Nat Rev Genet. 2009;10:295-304. 